\author{
含喹唑啉二酮片段的新型三酮类化合物的合成及生物活性

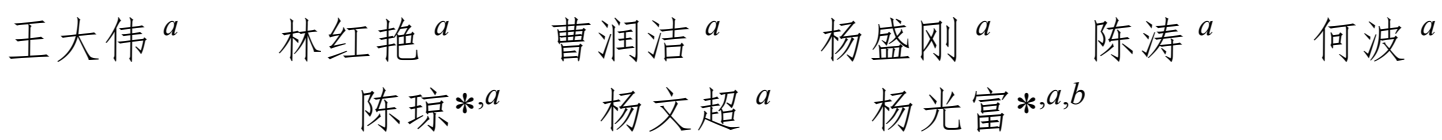 \\ ( ${ }^{a}$ 华中师范大学化学学院 教育部农药与化学生物学重点实验室 武汉 430079) \\ ${ }^{b}$ 天津化学化工协同创新中心 天津 300071 )
}

\begin{abstract}
摘要 对羟苯基丙酮酸双氧化酶(EC 1.13.11.27, HPPD)是一个重要的除草剂作用靶标. 为了寻找具有高效除草活性的 新型 HPPD 抑制剂, 在前期研究的基础上设计合成了 24 个含有喹唑啉二酮结构的三酮类化合物 9a $\sim 9 \mathbf{x}$. 所合成的化合 物均经过 ${ }^{1} \mathrm{H}$ NMR, ${ }^{13} \mathrm{C}$ NMR 和 HRMS 的表征. 以来源于拟南芥的 HPPD (AtHPPD) 为测试对象进行酶抑制活性篎选, 结果表明所合成的大部分化合物对 $A t \mathrm{HPPD}$ 均表现出了较好的抑制效果, 其中化合物 $9 \mathbf{i}$ 的 $K_{\mathrm{i}}$ 值为 $0.005 \mu \mathrm{mol} / \mathrm{L}$, 显著 优于商品化对照药剂硝磺草酮 $\left(K_{\mathrm{i}}=0.013 \mu \mathrm{mol} / \mathrm{L}\right)$. 进一步温室盆栽簰选结果表明, 多数化合物在 $150 \mathrm{~g}$ ai $/ \mathrm{ha}$ 的剂量下 对六种供试杂草中的至少一种表现出 $80 \%$ 以上的防效, 特别是化合物 $9 \mathrm{~g}$ 在 $37.5 \mathrm{~g}$ ai $/ \mathrm{ha}$ 的低剂量下对所测试的六种杂 草中的四种仍表现出了大于 $85 \%$ 的抑制效果, 同时 $9 \mathrm{~g}$ 在 $150 \mathrm{~g}$ ai/ha 的剂量下对水稻和小麦均表现出了很好的作物安 全性, 可以作为先导化合物供进一步深入研究.
\end{abstract}

关键词＼cjkstart对羟苯基丙酮酸双氧化酶；抑制剂; 喹唑啉二酮; 除草剂; 先导化合物

\title{
Synthesis and Bioactivity Studies of Triketone-Containing Quinazoline-2,4-dione Derivatives
}

\author{
Wang, Dawei ${ }^{a} \quad$ Lin, Hongyan $^{a} \quad$ Cao, Runjie ${ }^{a} \quad$ Yang, Shenggang $^{a}$ Chen, Tao $^{a}$ \\ $\mathrm{He}, \mathrm{Bo}^{a} \quad$ Chen, Qiong* ${ }^{*} \quad$ Yang, Wenchao ${ }^{a}$ Yang, Guangfu*,a,b \\ $\left({ }^{a}\right.$ Key Laboratory of Pesticide \& Chemical Biology of Ministry of Education, College of Chemistry, Central China Normal \\ University, Wuhan 430079) \\ $\left({ }^{b}\right.$ Collaborative Innovation Center of Chemical Science and Engineering, Tianjin 300071)
}

\begin{abstract}
Hydroxyphenylpyruvate dioxygenase (EC 1.13.11.27, HPPD) is an important enzyme in the catabolism of tyrosine, catalyzing the conversion of 4-hydroxyphenyl pyruvic acid (HPPA) into homogentisic acid (HGA), belonging to the 2-his-1-carboxylate facial triad family of non-heme iron(II) enzymes. As an important enzyme in regulating the biosynthesis of tocopherols and plastoquinone in plants, HPPD is an important target for herbicides discovery. Previously, we have found that triketone-containing quinazoline-2,4-dione motif can be used as a novel lead structure for herbicides discovery. In this continuous work, we synthesized a total number of 24 new triketone-containing quinazoline-2,4-dione derivatives. The new compounds 9a $\sim 9 \mathbf{x}$ were prepared by using 5-methyl-2-nitrobenzoic acid as the starting material, followed by oxidation, esterification and reduction reactions, the main intermediate dimethyl 4-aminoisophthalate could be obtained in a yield of $83 \%$, then by another six steps of reactions the target compounds could be smoothly synthesized. All the title compounds were characterized by ${ }^{1} \mathrm{H}$ NMR, ${ }^{13} \mathrm{C}$ NMR and HRMS spectrum data. To explore the biology activity of these compounds, their in vitro Arabidopsis thaliana HPPD (AtHPPD) inhibitory activity and in vivo herbicidal activity were evaluated. The results of $A t$ HPPD inhibitory experiments indicated that, most of the synthesized compounds showed "good" to "the excellent" HPPD-inhibiting activities. To our delight that, compound $9 \mathbf{i}$ with a $K_{\mathrm{i}}$ value of $0.005 \mu \mathrm{mol} / \mathrm{L}$ is about two times more potent than that of mesotrione $\left(K_{\mathrm{i}}=0.013 \mu \mathrm{mol} / \mathrm{L}\right)$. The results of greenhouse experiments showed that, most of the synthesized compounds displayed at least $80 \%$ inhibition against one of six weeds tested at the rate of $150 \mathrm{~g}$ ai/ha. To our surprise that, compound $9 \mathrm{~g}$ showed over $85 \%$ inhibition against four of six tested weeds even at a rate as low as $37.5 \mathrm{~g}$ ai/ha. In addition, it was also safe for rice and wheat by post-emergent application at the rate of $150 \mathrm{~g}$ ai/ha. Furthermore, we also obtained some structure-activity relationships, the SAR indicated that too electron-withdrawing groups in 3,5-positions of benzene ring (3-position of quinazoline-2,4-dione) were detrimental to activity, too sterically bulk groups at $\mathrm{R}^{4}$ were also detrimental to activity. Thus, compound $\mathbf{9 g}$ emerged as a new lead compound for herbicidal discovery.

Keywords 4-hydroxyphenylpyruvate dioxygenase; quinazoline-2,4-dione; herbicidal activity; bioactivity study; lead com-
\end{abstract}

* E-mail: qchen@mail.ccnu.edu.cn; gfyang@mail.ccnu.edu.cn; Tel.: 0086-027-67867800; Fax: 0086-027-67867141

Received November 22, 2014; published January 4, 2015.

Supporting information for this article is available free of charge via the Internet at http://sioc-journal.cn.

Project supported by the National Key Technologies R\&D Program of China (No. 2011BAE06B03) and the National Natural Science Foundation of China (Nos. 21372093, 21172091).

项目受国家 “十二五” 科技支撑计划(No. 2011BAE06B03)资助和国家自然科学基金面上项目(Nos. 21372093, 21172091)资助. 


\section{1 引言}

对羟苯基丙酮酸双氧化酶(EC 1.13.11.27, HPPD)是 一种非亚铁血红素氧化酶, 几乎存在于所有的需氧生物 体中 ${ }^{[1 \sim 3]}$. 它能够将酪氨酸代谢过程中所产生的对着基 苯丙酮酸(HPPA)催化转化成尿黑酸(HGA) (图 1). 在人 体内, 尿黑酸能够进一步转化为马来酰乙酰乙酸和延胡 索酰乙酰乙酸, 如果这两种物质代谢异常将会导致酪氨 酸 I 型血症的发生, 通过抑制 HPPD 的活性即能达到治 疗这种疾病的目的. 因此, 在医学领域 HPPD 是一个开 发药物的重要靶标. 目前, FDA 已经批准 HPPD 抑制剂 尼替西农(NTBC)用来治疗这种罕见的疾病. 在植物体 内, 尿黑酸能够进一步转化成质体醌和生育酚. 如果 HPPD 被抑制将会导致植物光合作用中电子链的传递受 阻, 进而导致植物体出现白化症状而死亡. 因此, 在农 业领域, HPPD 是开发除草剂的重要靶标 ${ }^{[4]}$. 虽然 HPPD 抑制型除草剂的使用历史可以追溯到 19 世纪 70 年代, 但直到 90 年代 HPPD 才被揭示是该类除草剂的分子靶 标. HPPD 抑制型除草剂具有杀草谱广、活性高、低毒、 作物选择性高以及对环境友好等一系列优点. 此外, 该 类除草剂还能够有效防治一些抗性杂草, 例如抗 ACCase、AHAS 抑制型除草剂的杂草、抗草甘膦的杂草. 正因为如此, 以 HPPD 为靶标设计合成新型除草剂已经 成为近年来农药化学研究的热点领域 ${ }^{[513]}$.<smiles>CC(C)CCCCCCC(=O)O</smiles>

L-Tyr<smiles>O=C(O)C(=O)c1ccc(O)cc1</smiles>

HPPA<smiles>O=C(O)Cc1cc(O)ccc1O</smiles>

HGA
图 1 HPPD 的催化反应

Figure 1 Catalytic reaction of HPPD

目前，已经报道的 HPPD 抑制剂可以分为四种结构 类型: 三酮类、吡唑类、异噁唑类 (二酮氧类 $)^{[6]}$ 以及其它 结构类型 ${ }^{[11,12]}$. 虽然 HPPD 抑制剂的化学结构差别比较 大, 但它们的分子结构具有以下共同特征: (1) 分子中 含有能够与二价铁进行配位的基团, 这些基团的最小结 构单元为 2-苯甲酰基-1-烯醇或者 2-杂环酰基-1-烯醇结 构; (2) 抑制剂经水解或者进入植物体内经代谢后能够 转化成含有特点(1)所述最小结构单元的化合物 ${ }^{[14 ~ 16]}$. 近年来, 专利上不断有新颖结构的 HPPD 抑制剂报道, 如含有吡啶酮结构的衍生物 ${ }^{[17]}$ 、含有氧代吡嗪结构的化 合物 ${ }^{[18]}$ 、含有 2-喹啉酮结构的衍生物 ${ }^{[19]}$ 以及含有嘧啶酮 结构的化合物 ${ }^{[20]}$. 这些新型的 HPPD 抑制剂均表现出了

优良的除草活性以及良好的作物选择性，其中部分化合 物已进入产业化前期开发研究.

我们课题组一直从事于具有生物活性小分子化合 物的设计, 合成以及生物活性研究, 并成功获得了多个

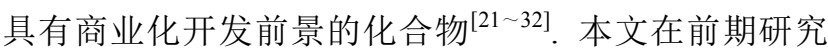
工作的基础上 ${ }^{[14,15]}$, 设计合成了含有喹唑啉二酮结构的 三酮类化合物，并对该类化合物的结构与活性关系进行 系统研究, 从而为进一步寻找具有更高除草和更广谱作 物选择性的除草先导化合物奠定基础(图 2).

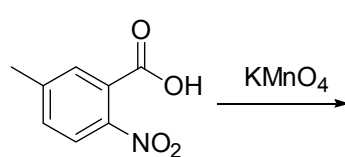

1<smiles>COC(=O)c1ccc([N+](=O)[O-])c(C(=O)OC)c1</smiles>

3

Substituted phenyl isocyanates Pyridine<smiles>O=C(O)c1ccc([N+](=O)[O-])c(C(=O)O)c1</smiles>
2<smiles>COC(=O)c1ccc(N)c(C(=O)OC)c1</smiles><smiles>COC(=O)c1ccc2[nH]c(=O)n(-c3ccccc3)c(=O)c2c1</smiles>

$\mathrm{Cs}_{2} \mathrm{CO}_{3}, \mathrm{CH}_{3} \mathrm{I}$ or $\mathrm{C}_{2} \mathrm{H}_{5} \mathrm{I}$ or Substituted benzyl bromides DMF<smiles>[R]#[R17]n1c(=O)n(-c2ccccc2)c(=O)c2cc(C(=O)OC)ccc21</smiles>

$\mathrm{HOAc}, \mathrm{H}_{2} \mathrm{SO}_{4}, \mathrm{H}_{2} \mathrm{O}$<smiles>[R1]n1c(=O)n(-c2cccc([R3]([H])([H])[H])c2)c(=O)c2cc(C(=O)O)ccc21</smiles>

$7 a \sim 7 q$

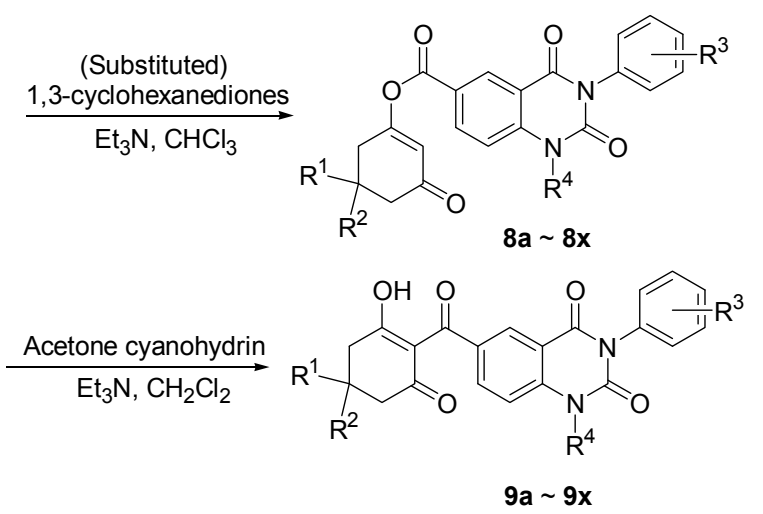

图 2 化合物 9a $\sim 9 \mathrm{x}$ 的合成路线

Figure 2 Synthetic routes of compounds $9 \mathbf{a} \sim 9 \mathbf{x}$ 


\section{2 结果与讨论}

\section{1 化合物的合成讨论}

从图 2 可以看出, 以 5-甲基 2-硝基苯甲酸为起始原 料, 经过 9 步反应即可制得化合物 9a $\sim 9 x$. 5-甲基 2-硝 基苯甲酸经过氧化、甲酯化以及还原反应即可制备关键 中间体 2-氨基间苯二甲酸甲酯 4 . 中间体 4 与含有不同 取代基的苯基异氧酸酯在吡啶溶液中反应, 即能以中等 偏上的收率制得中间体 $5 \mathrm{a} \sim 5 \mathbf{j} .5 \mathrm{a} \sim 5 \mathrm{j}$ 在碳酸铯的存在 下与碘甲烷、碘乙烷或者含有不同取代基的茮溴进行反 应, 即能以较高的收率合成化合物 $\mathbf{6 a} \sim \mathbf{6 q}$. 如果使用碳 酸钾或者碳酸钠, 反应难以进行, 通常需要在加热的条 件下反应 $24 \mathrm{~h}$ 以上才能够反应完全. 而使用碳酸铯, 反 应在室温条件下就能快速完成, 并且以较高的分离收率
得到 6a $\sim 6 q$. 此外, 我们还发现化合物 $6 a \sim 6 q$ 分子中 的喹唑啉二酮结构在碱性条件下(如氢氧化锂, 氢氧化 钠，氢(氧化钾)很容易开环，同时该化合物在碱性条件 下进行水解时, 反应收率较低并伴有大量的副产物生

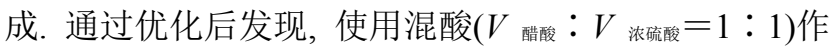
为水解试剂, $6 \mathbf{a} \sim 6 \mathbf{q}$ 即能以较高的收率水解得到 $7 \mathbf{a} \sim$ 7q. 7a 7q 在四氢呋喃溶剂中与氯化亚砜反应制得相应 的酰氯，无需纯化直接用于下一步反应，制得对应的烯 醇酯化合物 $8 \mathbf{a} \sim 8 \mathbf{x}$. 最后, 以丙酮氰醇作为催化剂，经 Fries 重排反应制得化合物 9a $\sim 9 x$.

\subsection{AtHPPD 酶抑制活性与构效关系}

表 1 列出了化合物 9a $\sim 9 x$ 对拟南芥 HPPD 的抑制 活性. 从中可以看出，绝大部分化合物对 $A t \mathrm{HPPD}$ 都表

表 1 化合物 9a 9x 苗后温室除草活性(抑制率\%)以及 $A t H P P D$ 酶活性抑制测试实验结果

Table 1 Post-emergent herbicidal activity (inhibition rating, $0 \sim 100$ ) of compounds $9 \mathbf{a} \sim \mathbf{9 x}$ and their inhibitory activities against $A t$ HPPD

\begin{tabular}{|c|c|c|c|c|c|c|c|c|c|c|c|c|}
\hline Compd. & $\mathrm{R}^{1}$ & $\mathrm{R}^{2}$ & $\mathrm{R}^{3}$ & $\mathrm{R}^{4}$ & $\begin{array}{c}\text { 剂量 } \\
\text { (g ai/ha) }\end{array}$ & 苟麻 & 鳢肠 & 反枝苋 & 稗草 & 马唐 & 狗尾草 & $\begin{array}{c}K_{\mathrm{i}} \text { for } A t \mathrm{HPPD} / \\
(\mu \mathrm{mol} / \mathrm{L})\end{array}$ \\
\hline \multirow{3}{*}{$9 a$} & \multirow{3}{*}{$\mathrm{H}$} & \multirow{3}{*}{$\mathrm{CH}_{3}$} & \multirow{3}{*}{$\mathrm{H}$} & \multirow{3}{*}{$\mathrm{CH}_{3}$} & 150 & 50 & 60 & 87.5 & 90 & 95 & 80 & \multirow{3}{*}{$0.028 \pm 0.001$} \\
\hline & & & & & 75 & 15 & 30 & 75 & 80 & 90 & 50 & \\
\hline & & & & & 37.5 & 0 & 0 & 70 & 75 & 80 & 30 & \\
\hline $9 b$ & $\mathrm{H}$ & $\mathrm{H}$ & $4-\mathrm{CH}\left(\mathrm{CH}_{3}\right)_{2}$ & $\mathrm{CH}_{3}$ & 150 & 50 & 0 & 35 & 25 & 100 & 50 & $0.034 \pm 0.003$ \\
\hline \multirow[t]{2}{*}{$9 c$} & \multirow[t]{2}{*}{$\mathrm{H}$} & \multirow[t]{2}{*}{$\mathrm{H}$} & \multirow[t]{2}{*}{ 2,4,6-tri- $\mathrm{CH}_{3}$} & \multirow[t]{2}{*}{$\mathrm{CH}_{3}$} & 75 & 95 & 65 & 50 & 87.5 & 90 & 90 & \multirow[t]{2}{*}{$0.043 \pm 0.002$} \\
\hline & & & & & 37.5 & 85 & 50 & 45 & 85 & 82.5 & 80 & \\
\hline 9d & $\mathrm{CH}_{3}$ & $\mathrm{CH}_{3}$ & $2-\mathrm{CF}_{3}$ & $\mathrm{CH}_{3}$ & 150 & 80 & 50 & 80 & 40 & 85 & 45 & $0.150 \pm 0.03$ \\
\hline $9 e$ & $\mathrm{H}$ & $\mathrm{H}$ & $2-\mathrm{Cl}-5-\mathrm{CF}_{3}$ & $\mathrm{CH}_{3}$ & 150 & 100 & 0 & 70 & 65 & 40 & 80 & $0.045 \pm 0.002$ \\
\hline 9f & $\mathrm{CH}_{3}$ & $\mathrm{CH}_{3}$ & $2-\mathrm{Cl}-5-\mathrm{CF}_{3}$ & $\mathrm{CH}_{3}$ & 150 & 65 & 20 & 0 & 0 & 25 & 10 & $0.195 \pm 0.092$ \\
\hline \multirow[t]{2}{*}{$9 \mathrm{~g}$} & \multirow[t]{2}{*}{$\mathrm{H}$} & \multirow[t]{2}{*}{$\mathrm{H}$} & \multirow[t]{2}{*}{$2-\mathrm{CH}_{3}-6-\mathrm{Cl}$} & \multirow[t]{2}{*}{$\mathrm{CH}_{3}$} & 75 & 97.5 & 50 & 92.5 & 80 & 95 & 100 & \multirow[t]{2}{*}{$0.027 \pm 0.003$} \\
\hline & & & & & 37.5 & 90 & 40 & 65 & 85 & 90 & 95 & \\
\hline \multirow{3}{*}{$9 \mathrm{~h}$} & \multirow{3}{*}{$\mathrm{CH}_{3}$} & \multirow{3}{*}{$\mathrm{CH}_{3}$} & & & 150 & 100 & 75 & 90 & 90 & 90 & 92.5 & \\
\hline & & & $2-\mathrm{CH}_{3}-6-\mathrm{Cl}$ & $\mathrm{CH}_{3}$ & 75 & 100 & 65 & 87.5 & 85 & 85 & 85 & $0.060 \pm 0.006$ \\
\hline & & & & & 37.5 & 95 & 50 & 82.5 & 80 & 75 & 70 & \\
\hline $9 \mathrm{i}$ & $\mathrm{H}$ & $\mathrm{H}$ & $2-\mathrm{CH}_{3}-4-\mathrm{NO}_{2}$ & $\mathrm{CH}_{3}$ & 150 & 0 & 0 & 85 & 0 & 30 & 70 & $0.005 \pm 0.001$ \\
\hline $9 \mathbf{j}$ & $\mathrm{CH}_{3}$ & $\mathrm{CH}_{3}$ & $2-\mathrm{CH}_{3}-4-\mathrm{NO}_{2}$ & $\mathrm{CH}_{3}$ & 150 & 0 & 80 & 70 & 0 & 30 & 0 & $0.065 \pm 0.012$ \\
\hline $9 k$ & $\mathrm{H}$ & $\mathrm{H}$ & $3,5-\mathrm{di}-\mathrm{CF}_{3}$ & $\mathrm{CH}_{3}$ & 150 & 0 & 0 & 60 & 0 & 0 & 0 & $0.183 \pm 0.009$ \\
\hline 91 & $\mathrm{H}$ & $\mathrm{H}$ & 3,5 -di- $\mathrm{CF}_{3}$ & $\mathrm{CH}_{2} \mathrm{CH}_{3}$ & 150 & 0 & 70 & 30 & 0 & 0 & 0 & $0.139 \pm 0.006$ \\
\hline $9 m$ & $\mathrm{H}$ & $\mathrm{H}$ & 3,5-di-F & $\mathrm{CH}_{3}$ & 150 & 30 & 75 & 80 & 75 & 15 & 60 & $0.038 \pm 0.002$ \\
\hline $9 n$ & $\mathrm{CH}_{3}$ & $\mathrm{CH}_{3}$ & 3,5-di-F & $\mathrm{CH}_{3}$ & 150 & 0 & 75 & 75 & 0 & 0 & 0 & $0.052 \pm 0.001$ \\
\hline $9 p$ & $\mathrm{H}$ & $\mathrm{H}$ & $3-\mathrm{F}$ & $\mathrm{CH}_{2} \mathrm{CH}_{3}$ & 150 & 0 & 95 & 85 & 75 & 15 & 65 & $0.017 \pm 0.003$ \\
\hline $\mathbf{9 q}$ & $\mathrm{CH}_{3}$ & $\mathrm{CH}_{3}$ & $3-\mathrm{F}$ & $\mathrm{CH}_{2} \mathrm{CH}_{3}$ & 150 & 30 & 90 & 85 & 65 & 30 & 0 & $0.043 \pm 0.002$ \\
\hline $9 r$ & $\mathrm{H}$ & $\mathrm{H}$ & $3-\mathrm{F}$ & $\mathrm{CH}_{2}-3-\mathrm{OCH}_{3}-\mathrm{C}_{6} \mathrm{H}_{4}$ & 150 & 0 & 0 & 0 & 0 & 0 & 0 & $0.410 \pm 0.027$ \\
\hline $9 \mathrm{~s}$ & $\mathrm{H}$ & $\mathrm{H}$ & $3-\mathrm{F}$ & $\mathrm{CH}_{2}-3-\mathrm{F}-\mathrm{C}_{6} \mathrm{H}_{4}$ & 150 & 0 & 15 & 30 & 0 & 0 & 0 & $0.216 \pm 0.010$ \\
\hline $9 t$ & $\mathrm{CH}_{3}$ & $\mathrm{CH}_{3}$ & $3-\mathrm{F}$ & $\mathrm{CH}_{2}-3-\mathrm{F}-\mathrm{C}_{6} \mathrm{H}_{4}$ & 150 & 30 & 0 & 0 & 0 & 0 & 0 & $>2$ \\
\hline $9 u$ & $\mathrm{H}$ & $\mathrm{H}$ & $3-\mathrm{F}$ & $\mathrm{CH}_{2}-4-\mathrm{OCF}_{3}-\mathrm{C}_{6} \mathrm{H}_{4}$ & 150 & 0 & 0 & 0 & 0 & 0 & 0 & $0.190 \pm 0.015$ \\
\hline $9 v$ & $\mathrm{CH}_{3}$ & $\mathrm{CH}_{3}$ & $3-\mathrm{F}$ & $\mathrm{CH}_{2}-4-\mathrm{OCF}_{3}-\mathrm{C}_{6} \mathrm{H}_{4}$ & 150 & 0 & 0 & 0 & 0 & 0 & 0 & $0.414 \pm 0.003$ \\
\hline
\end{tabular}




\begin{tabular}{|c|c|c|c|c|c|c|c|c|c|c|c|c|}
\hline Compd. & $\mathrm{R}^{1}$ & $\mathrm{R}^{2}$ & $\mathrm{R}^{3}$ & $\mathrm{R}^{4}$ & $\begin{array}{c}\text { 剂量 } \\
\text { (g ai/ha) }\end{array}$ & 苟麻 & 鳢肠 & 反枝苋 & 稗草 & 马唐 & 狗尾草 & $\begin{array}{c}K_{\mathrm{i}} \text { for } A t \mathrm{HPPD} / \\
(\mu \mathrm{mol} / \mathrm{L})\end{array}$ \\
\hline $9 w$ & $\mathrm{H}$ & $\mathrm{H}$ & $3-\mathrm{F}$ & $\mathrm{CH}_{2}-3-\mathrm{CF}_{3}-\mathrm{C}_{6} \mathrm{H}_{4}$ & 150 & 0 & 0 & 0 & 0 & 0 & 0 & $0.414 \pm 0.044$ \\
\hline \multirow[t]{4}{*}{$9 \mathrm{x}$} & $\mathrm{H}$ & $\mathrm{H}$ & $3-\mathrm{F}$ & $\mathrm{CH}_{2}-3,5$-di-F- $\mathrm{C}_{6} \mathrm{H}_{4}$ & 150 & 0 & 0 & 0 & 0 & 0 & 0 & $0.409 \pm 0.010$ \\
\hline & & & & & 150 & 100 & 100 & 100 & 85 & 95 & 20 & \\
\hline & & & Mesotrione & & 75 & 100 & 100 & 100 & 75 & 60 & 0 & $0.013 \pm 0.001$ \\
\hline & & & & & 37.5 & 100 & 100 & 100 & 30 & 30 & 0 & \\
\hline
\end{tabular}

现出了较好的抑制活性. 例如, 化合物 90 的 $K_{\mathrm{i}}$ 值为 $0.013 \mu \mathrm{mol} / \mathrm{L}$, 与对照药剂硝磺草酮 $\left(K_{\mathrm{i}}=0.013 \mu \mathrm{mol} / \mathrm{L}\right)$ 相当. 化合物 $9 \mathrm{i}$ 的 $K_{\mathrm{i}}$ 值为 $0.005 \mu \mathrm{mol} / \mathrm{L}$, 比硝磺草酮低 1 倍. 通过分析化合物 9i 与 $A t \mathrm{HPPD}$ 的结合模式(图 3, A), 我们发现 9i 与 $A t H P P D$ 形成了三个比较重要的相互 作用. 一是 $9 \mathbf{i}$ 分子中的三酮部分能与 $A t \mathrm{HPPD}$ 活性中心 的二价铁形成双齿螯合的配位作用; 二是 $9 \mathbf{i}$ 分子中的喹 唑啉二酮环与 $A t H P P D$ 活性口袋中的第 403 位的苯丙氨 酸(Phe403)和第 360 位的苯丙氨酸(Phe360)形成了三明 治型的 $\pi-\pi$ 相互作用; 三是 $9 \mathbf{i}$ 分子的硝基氧原子与第 272 位谷氨酰胺 $(G \ln 272)$ 的 NH 形成了距离为 $3.1 \AA$ 的 $\mathrm{H}$ 键相互作用. 分析表 1 中的结果, 可以归纳出化合物结 构与 $A t \mathrm{HPPD}$ 抑制活性之间具有如下关系: (1)如果在喹 唑啉二酮 3 位苯环上同时引入一个供电子基和一个吸电 子基, 往往有助于其酶活抑制效果的提高, 如 $9 \mathrm{i}$ 与 $9 \mathrm{~g}$ 的活性优于 9e; (2) 如果喹唑啉二酮 3 位苯环的 2 个间 位都被取代时, 强吸电子基的存在对其酶抑制活性的提 高是不利的, 如 $9 \mathrm{~m}$ 的酶抑制活性优于 $9 k$; (3)如果将 $\mathrm{R}^{4}$ 的取代基由甲基换成乙基, 将有助于其活性的提高, 如 91 的酶抑制活性优于 9k, 90 的酶抑制活性优于 9n; (4) 如果在 $\mathrm{R}^{4}$ 引入空间位阻较大的取代基(如 9r 9x) 将会 导致化合物酶抑制活性的明显降低. 为了解释这一原 因, 我们以代表性化合物 9t 为例, 采用分子模拟技术研 究了其与 $A t \mathrm{HPPD}$ 的相互作用. 结果显示, 由于 $9 \mathrm{t}$ 分子 中间氟苄基的体积较大, 使得它与 $A t \mathrm{HPPD}$ 的第 347 位
亮氨酸(Leu 347)残基的 2 个甲基的距离比较近(分别是 $3.1 \AA$ 和 $3.0 \AA$ ), 这样就会产生较强的空间排斥作用. 同 时, 9t 分子中环已二酮 5 位上的甲基与第 261 位苏氨酸 (Thr 261)的距离为 $3.0 \AA$, 同样也产生了较强的空间排 斥作用，从而导致这些化合物的活性下降.

\section{3 除草活性与构效关系}

我们对所合成的化合物进行了温室盆栽除草活性 笁选, 结果见表 1 . 从中可以看出, 在 $150 \mathrm{~g}$ ai/ha 的剂量 下，大部分化合物对六种杂草中的至少一种表现出 $80 \%$ 以上的防效. 特别值得指出的是, 将剂量降低至 $37.5 \mathrm{~g}$ ai/ha 时，化合物 9g 对苟麻、稗草、马唐和狗尾草的防 效依然维持在 $85 \%$ 以上，同时对鳢肠和反枝苋的防效分 别为 $40 \%$ 和 $65 \%$ ，与硝磺草酮相比，明显提高了对禾本 科杂草的防效. 初步总结化合物除草活性与结构之间的 关系: (1) 如果喹唑啉二酮 3 位苯环上的取代基 $\mathrm{R}^{3}$ 为给 电子基或者是弱的吸电子基时，化合物往往会具有较好 的除草活性, 如化合物 9a, 9c, 9g. (2) 如果在喹唑啉二 酮 3 位苯环的邻位引入一个甲基或者氯原子, 同时在 4 位或者 5 位引入一个强吸电子基时，将会导致化合物活 性的降低，如化合物 9e 和 9i. (3) 如果在喹唑啉二酮 3 位 苯环的间位同时引入 2 个强吸电子基团将会导致化合物 活性的降低甚至丧失，如化合物 9k 与 9m. (4) 如果增大 取代基 $\mathrm{R}^{4}$ 的空间位阻将会导致化合物除草活性的丧失, 例如化合物 9r 基本上对所测试的六种杂草均无防效.

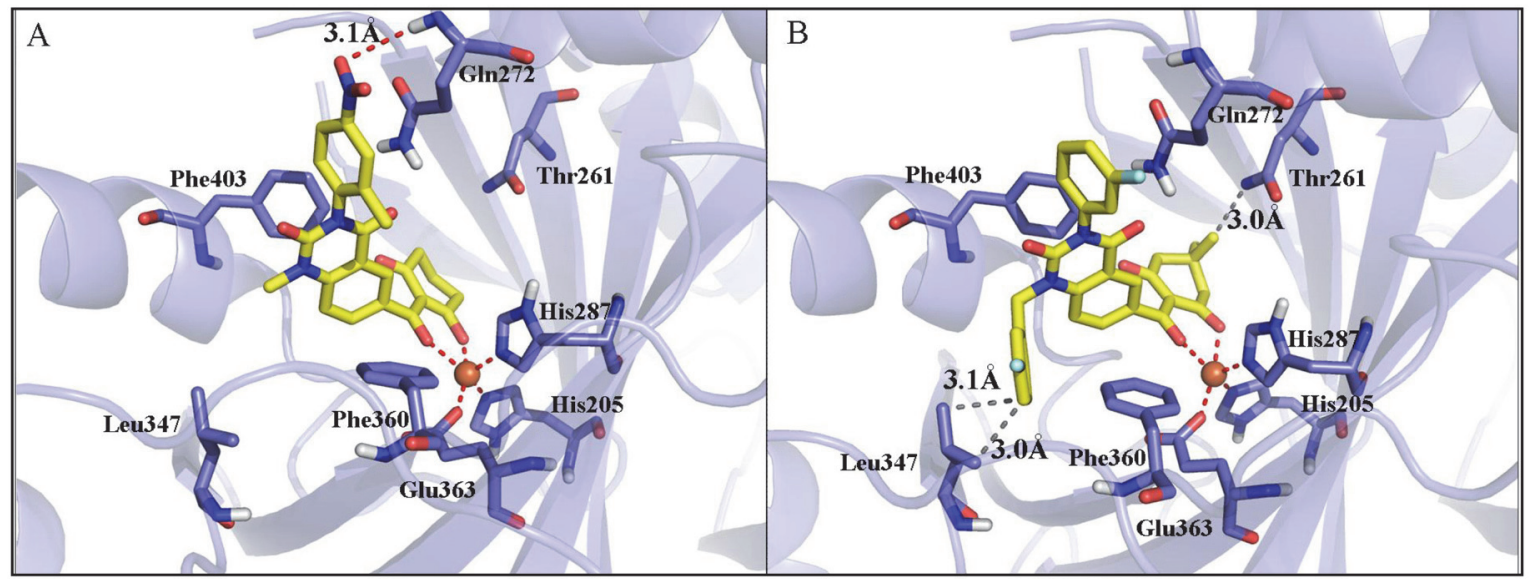

图 3 化合物 $9 \mathrm{i}(\mathrm{A})$ 和 $9 \mathrm{t}(\mathrm{B})$ 与 $A t \mathrm{HPPD}$ 的模拟结合模式

Figure 3 Simulated binding modes of compounds $9 i$ (A) and $9 t$ (B) with $A t \mathrm{HPPD}$ 


\section{4 化合物除草活性与 AtHPPD 酶抑制活性之间的关} 系

从表 1 可以看出, 化合物 9a $\sim 9 \mathbf{x}$ 对 $A t \mathrm{HPPD}$ 的抑制 活性与其除草活性之间有着良好的相关性. 通常情况 下, 具有较高酶抑制活性的化合物, 往往表现出较好的 除草活性，这表明化合物发挥除草活性的机制是抑制了 杂草体内的 HPPD 的活性. 当然, 个别表现出较高酶抑 制活性的化合物(9i 和 9o) 并没有表现出理想的除草活 性, 这可能是因为这些化合物的物理化学性质不太理 想, 不能很好地被植物体吸收传导. 因此, 在今后的分 子设计中, 优化抑制剂的物理化学性质使之具有更好的 类农药性质是需要加以关注的重点.

\section{5 作物选择性测试}

我们以玉米、大豆、棉花、水稻、大麦和油菜六种 作物为对象, 选择表现出良好除草活性的化合物 $9 \mathrm{~g}$ 开 展了进一步的作物安全性试验. 结果发现, 在 $150 \mathrm{~g}$ ai/ha 的剂量下, $9 \mathrm{~g}$ 对水稻和小麦均表现出了良好的作物 安全性. 相反, 在同样剂量下, 商品化除草剂硝磺草酮 仅对玉米表现出了较好的安全性, 对其他五种作物均没 有表现出安全性. 值得指出的是, 同时对水田作物和旱 田作物具有安全性的除草剂具有非常重要的应用前景. 综合酶抑制活性、温室除草活性以及作物安全性试验结 果, 我们认为化合物 $9 \mathrm{~g}$ 具备开发成为玉米和水稻田除 草剂的潜力, 值得进一步深入研究.

\section{3 结论}

本文设计合成了 24 个结构新颖的含喹唑啉二酮结 构单元的 HPPD 抑制剂, 这些化合物对来源于拟南芥的 HPPD 具有较好的抑制活性, 其中化合物 $9 \mathbf{i}\left(K_{\mathrm{i}}=0.005\right.$ $\mu \mathrm{mol} / \mathrm{L}$ )对 $A t \mathrm{HPPD}$ 的抑制活性是商品化除草剂硝磺草 酮 $\left(K_{\mathrm{i}}=0.013 \mu \mathrm{mol} / \mathrm{L}\right)$ 的 2 倍. 进一步开展温室盆栽除草 活性篮选和作物安全性试验, 发现化合物 $9 \mathrm{~g}\left(K_{\mathrm{i}}=0.027\right.$ $\mu \mathrm{mol} / \mathrm{L}$ ) 在 $37.5 \mathrm{~g}$ ai/ha 的低剂量下仍然表现出广谱的除 草活性, 在 $150 \mathrm{~g}$ ai/ha 的剂量下对水稻和小麦均表现出 了较好的作物安全性, 表现出进一步深入研究的潜力.

\section{4 实验部分}

\section{1 仪器与试剂}

${ }^{1} \mathrm{H}$ NMR, ${ }^{13} \mathrm{C}$ NMR 用美国 VARIAN Mercury-Plus $400 \mathrm{MHz}$ 或 $600 \mathrm{MHz}$ 核磁共振仪 (TMS 为内标, DMSO- $d_{6}, \mathrm{CDCl}_{3}$ 或者 $\mathrm{CD}_{2} \mathrm{Cl}_{2}$ 为溶剂)测定; 高分辨质谱 用美国安捷伦公司生产的 Agilent 6224 TOF LC/MS 液 质联用仪测定. 常规柱层析硅胶和 TLC 硅胶板, 由青岛 海洋化工厂有限公司生产. 熔点用瑞士 Buchi B-545 数 字熔点仪 (温度计未较定) 测定. 实验中所用到的药品若 无特殊说明均可从试剂公司(如 Alfa Aesar 和 J\&K chemical)购买, 实验中所用到的溶剂在实验前均经过重
蒸或者干燥处理. 实验中使用的石油醚的沸程为 $60 \sim$ $90{ }^{\circ} \mathrm{C}$.

\section{2 合成方法}

\subsubsection{4-硝基间苯二甲酸 2 的合成}

将 5-甲基-2-硝基苯甲酸 1 (100 g, $553 \mathrm{mmol})$ 加入 到 $2500 \mathrm{~mL}$ 的三颈瓶中, 装上回流冷凝管并加入 1000 $\mathrm{mL}$ 水, 搅拌下加入 $\mathrm{KOH}(31 \mathrm{~g}, 553 \mathrm{mmol})$, 并加热到 $90{ }^{\circ} \mathrm{C}$. 待反应瓶中固体完全溶解后, 在 $1 \mathrm{~h}$ 内分批将 $\mathrm{KMnO}_{4}(262.2 \mathrm{~g}, 1659 \mathrm{mmol})$ 加入到反应瓶中, 加入完 毕后将体系继续在 $90{ }^{\circ} \mathrm{C}$ 反应 $3 \mathrm{~h}$. 反应完毕后趁热抽 滤, 用热水洗涤滤饼. 滤液在冰水浴中用浓 $\mathrm{HCl}$ 酸化, 调 $\mathrm{pH}$ 值为 $1 \sim 2$, 静置析出大量固体. 抽滤, 水洗, 干 燥, 得白色固体. 产量 105 g, 产率 90\%; m.p. 244 $246{ }^{\circ} \mathrm{C}$; ${ }^{1} \mathrm{H}$ NMR (600 MHz, DMSO- $\left.d_{6}\right) \delta$ : 13.99 (brs, $2 \mathrm{H}), 8.34(\mathrm{~d}, J=1.2 \mathrm{~Hz}, 1 \mathrm{H}), 8.27(\mathrm{dd}, J=8.4,1.8 \mathrm{~Hz}$, $1 \mathrm{H}), 8.08(\mathrm{~d}, J=8.4 \mathrm{~Hz}, 1 \mathrm{H})$.

\subsubsection{4-硝基间苯二甲酸甲酯 3 的合成}

将 4-硝基间苯二甲酸 2 (100 g, $474 \mathrm{mmol})$, 甲醇 $(500 \mathrm{~mL})$ 加入到 $1500 \mathrm{~mL}$ 三颈瓶中, 搅拌下缓慢滴加浓 $\mathrm{H}_{2} \mathrm{SO}_{4}(30 \mathrm{~mL})$, 滴加完毕后加热到回流, 并反应过夜. 待反应完毕后, 减压除去甲醇. 冷却后, 向反应瓶中加 入乙酸乙酯 $(800 \mathrm{~mL})$, 分别用水 $(200 \mathrm{~mL} \times 2)$, 饱和碳酸 钠溶液 $(200 \mathrm{~mL} \times 2)$, 饱和食盐水 $(200 \mathrm{~mL})$ 洗涤. 有机层 用无水 $\mathrm{Na}_{2} \mathrm{SO}_{4}$ 干燥, 脱干溶剂得白色结晶产品. 产量 107.6 g, 产率 95\%, m.p. $84 \sim 86{ }^{\circ} \mathrm{C} ;{ }^{1} \mathrm{H}(600 \mathrm{MHz}$, $\left.\mathrm{CDCl}_{3}\right) \delta: 8.44(\mathrm{~s}, 1 \mathrm{H}), 8.29(\mathrm{~d}, J=8.4 \mathrm{~Hz}, 1 \mathrm{H}), 7.93(\mathrm{~d}$, $J=8.4 \mathrm{~Hz}, 1 \mathrm{H}), 3.99$ (s, 3H), 3.95 (s, 3H).

\subsubsection{4-氨基间苯二甲酸甲酯 4 的制备}

将 4-硝基间苯二甲酸甲酯 $3(100 \mathrm{~g}, 419 \mathrm{mmol})$ 溶于 $800 \mathrm{~mL}$ 乙酸乙酯中, 搅拌下加入 $10 \mathrm{~g}$ 质量百分数为 $10 \%$ 的 $\mathrm{Pd} / \mathrm{C}$. 通入 $\mathrm{H}_{2}$, 并缓慢加热到 $40{ }^{\circ} \mathrm{C}$, 反应约 20 $\mathrm{h}, \mathrm{TLC}$ 跟踪反应进程[展开剂: $V$ (石油醚 $) / V($ 乙酸乙酯 $)=$ 5/1]至原料消失. 反应完毕后, 停止加热, 将反应液冷 却到室温并过滤除去反应中所添加的 $\mathrm{Pd} / \mathrm{C}$. 减压除去 滤液中的溶剂得白色结晶固体 $84.8 \mathrm{~g}$, 产率 97\%, m.p. $127 \sim 129{ }^{\circ} \mathrm{C} .{ }^{1} \mathrm{H}\left(400 \mathrm{MHz}, \mathrm{CDCl}_{3}\right) \delta: 8.59$ (d, $J=1.6$ $\mathrm{Hz}, 1 \mathrm{H}), 7.91(\mathrm{dd}, J=8.4,2.0 \mathrm{~Hz}, 1 \mathrm{H}), 6.66(\mathrm{~d}, J=8.8 \mathrm{~Hz}$, 1H), 6.28 (brs, 2H), 3.90 (s, 3H), 3.88 (s, 3H).

4.2.4 中间体(3-取代苯基-1- $H$ 喹唑啉-2,4 $(1 H, 3 H)$-二 酮)-6-羧酸甲酯 $5 \mathbf{a} \sim 5 \mathbf{j}$ 的制备

将 4-氨基间苯二甲酸甲酯 4 (20 mmol), 吡啶 (30 $\mathrm{mL}$ )加入到 $100 \mathrm{~mL}$ 的两颈瓶中, 搅拌下加入取代的苯 基异氧酸酯 $(25 \mathrm{mmol})$, 氮气保护下 $100{ }^{\circ} \mathrm{C}$ 反应 $6 \mathrm{~h}$. TLC 跟踪反应进程[展开剂: $V$ (石油醚) $/ V$ (乙酸乙酯 $)=$ 4/1], 反应完毕后, 冷却到室温并将反应液倒入 $100 \mathrm{~mL}$ 水中, 混合液剧烈搅拌 $30 \mathrm{~min}$, 析出大量白色沉淀, 抽 滤，固体用乙梄洗涤，干燥后即得 $\mathbf{5 a} \sim \mathbf{5 j}$. 
中间体 $\mathbf{5} \mathbf{a} \sim \mathbf{5} \mathbf{j}$ 的结构表征数据见支持信息.

4.2.5 中间体(3-取代苯基-1-取代喹唑啉-2,4(1H,3H)-二 酮)-6-羧酸甲酯 $6 \mathrm{a} \sim 6 \mathbf{q}$ 的制备

将中间体 $\mathbf{5 a} \sim 5 \mathbf{j}$ (15 mmol), $N, N$-二甲基甲酰胺 (75 $\mathrm{mL}$ )加入到 $200 \mathrm{~mL}$ 单颈瓶中, 搅拌下加入 $\mathrm{Cs}_{2} \mathrm{CO}_{3}$ (18 $\mathrm{mmol})$. 室温下搅拌 $30 \mathrm{~min}$ 后, 缓慢滴加碘甲烷或者碘 乙烷 $(30 \mathrm{mmol})$, 滴加完毕后室温下继续搅拌反应 $12 \mathrm{~h}$. TLC 跟踪反应进程[展开剂: $V$ (石油醚 $) / V$ (丙䣶 $)=3 / 1$ ], 反应完毕后将反应液倒入 $100 \mathrm{~mL}$ 水中, 剧烈搅拌 30 $\mathrm{min}$, 所得固体抽滤, 干燥后即得 $\mathbf{6 a} \sim \mathbf{6}$. 将中间体 $\mathbf{5 j}$ (15 mmol), $N, N$-二甲基甲酰胺 $(75 \mathrm{~mL}$ )加入到 $200 \mathrm{~mL}$ 单颈瓶中, 搅拌下加入 $\mathrm{Cs}_{2} \mathrm{CO}_{3}(18 \mathrm{mmol})$. 室温下搅拌 $30 \mathrm{~min}$ 后, 缓慢滴加取代的苄溴 $(18 \mathrm{mmol})$, 滴加完毕后 室温下继续摚拌 $12 \mathrm{~h}$. TLC 跟踪反应进程[展开剂: $V$ (石 油醚 $) / V$ (丙䣶 $)=3 / 1$, 反应完毕后将反应液倒入 $100 \mathrm{~mL}$ 水中, 剧烈搅拌 $30 \mathrm{~min}$, 所得固体抽滤, 干燥后即得 $\mathbf{6 m} \sim 6 \mathbf{6 q}$.

中间体 $\mathbf{6 a} \sim \mathbf{6 q}$ 的结构表征数据见支持信息.

4.2.6中间体(3-取代苯基-1-取代喹唑啉-2,4 (1H,3H)二酮)-6-羧酸 $7 \mathbf{a} \sim 7 \mathbf{q}$ 的制备

将中间体 $\mathbf{6 a} \sim \mathbf{6 q}(15 \mathrm{mmol})$, 冰醋酸 $(50 \mathrm{~mL})$ 和水 $(50 \mathrm{~mL})$ 加入到 $500 \mathrm{~mL}$ 单颈瓶中. 搅拌下缓慢滴加浓 $\mathrm{H}_{2} \mathrm{SO}_{4}(50 \mathrm{~mL})$, 滴加完毕后, 加热到 $100{ }^{\circ} \mathrm{C}$ 反应 $12 \mathrm{~h}$. TLC 跟踪反应历程[展开剂: $V$ (石油醚) $/ V$ (丙酮 $)=3 / 1$ ], 反应完毕后, 将体系冷却到室温并倒入 $200 \mathrm{~mL}$ 冰水中. 剧烈搅拌 $30 \mathrm{~min}$, 所得固体抽滤, 干燥后即得 $7 \mathbf{a} \sim 7 \mathbf{q}$.

中间体 $7 \mathbf{a} \sim 7 \mathbf{q}$ 的结构表征数据见支持信息.

4.2.7中间体 3-((3-取代苯基-1-取代喹唑啉-2,4（1H, $3 H$ )-二酮)-6-甲酰基)-2-(取代)环已烯基-1-酮 $8 \mathbf{a} \sim 8 \mathbf{x}$ 的 制备

将中间体 7a $\sim$ q $(2 \mathrm{mmol})$, 四氢呋喃 $(40 \mathrm{~mL})$ 加 入到 $100 \mathrm{~mL}$ 单颈瓶中, 搅拌下缓慢滴加氯化亚砜(3 $\mathrm{mmol}$ ), 滴加完毕后再加入 2 滴 $N, N$-二甲基甲酰胺. 将 反应体系加热到 $75{ }^{\circ} \mathrm{C}$ 回流反应 $3 \mathrm{~h}$. 反应结束后脱干溶 剂即得到对应的酰氯, 将所制得的酰氯溶于 $20 \mathrm{~mL}$ 氯 仿. 将上述溶液于 $0{ }^{\circ} \mathrm{C}$ 下缓慢滴加到由 1,3 -环已二酮 $(2 \mathrm{mmol}), \mathrm{Et}_{3} \mathrm{~N}(4 \mathrm{mmol})$ 和氯仿 $(20 \mathrm{~mL})$ 组成的溶液中. 滴加完毕后在 $0{ }^{\circ} \mathrm{C}$ 下继续搅拌反应 $1 \mathrm{~h}$, TLC 跟踪反应 历程[展开剂: $V$ (石油醚 $) / V$ (丙酮 $)=5 / 2$ ]. 反应完毕后, 有 机层分别用水 $(20 \mathrm{~mL})$ 洗涤一次, $1 \mathrm{~mol} / \mathrm{L}$ 的盐酸溶液洗 涤 $(20 \mathrm{~mL} \times 2)$, 饱和碳酸氢钠溶液 $(20 \mathrm{~mL} \times 2)$ 洗涤, 无 水 $\mathrm{Na}_{2} \mathrm{SO}_{4}$ 干燥, 脱溶, 柱层析 [洗脱剂: $V$ (石油醚) $/ V$ (丙 酥 $)=5 / 1$ ] 后即得 $\mathbf{8 a} \sim \mathbf{8 x}$.

中间体 $\mathbf{8 a} \sim \mathbf{8 x}$ 的结构表征数据见支持信息.
4.2.8 目标化合物 3-((3-取代苯基-1-取代喹唑啉-2,4 (1H,3H)-二酮)-6-甲酰基)(取代)环已烷-1,3-二酮 9a〜9x 的制备

将中间体 $\mathbf{8 a} \sim \mathbf{8 x}(1 \mathrm{mmol}), \mathrm{CH}_{2} \mathrm{Cl}_{2}(30 \mathrm{~mL})$ 加入到 $100 \mathrm{~mL}$ 反应瓶中, 搅拌下加入 $\mathrm{Et}_{3} \mathrm{~N}(2 \mathrm{mmol})$, 丙酮氰 醇(0.1 mmol), 室温下反应 $20 \mathrm{~h}, \mathrm{TLC}$ 跟踪[展开剂: $V$ (石 油醚 $) / V($ 丙酮 $)=5 / 2$ ]反应至原料消失. 反应完毕后，有 机层分别用 $1 \mathrm{~mol} / \mathrm{L}$ 的盐酸溶液 $(30 \mathrm{~mL} \times 3)$, 饱和食盐 水 $(30 \mathrm{~mL})$ 洗涤, 无水硫酸钠干燥, 减压脱去溶剂, 所得 油状物用 $10 \mathrm{~mL}$ 甲醇重结晶得化合物 $\mathbf{9 a} \sim 9 \mathbf{x}$.

目标化合物 9a 9x 的结构表征数据见支持信息.

\section{3 目标化合物 9a 9x 的除草活性测试}

所合成的目标化合物委托国家南方农药创制中心 浙江省化工研究院生测安评中心测定了对六种杂草: 苟 麻 Abutilon juncea, 鳢肠 Eclipta prostrate, 反枝苋 Amaranthus retroflexu, 稗草 Echinochloa crusgalli，马唐 Digitaria sanguinalis, 狗尾草 Setaria faberii 的抑制效果. 所测试的方法采用的是盆栽法: 取内径 $6 \mathrm{~cm}$ 花盆, 装复 合土 $[V($ 菜园土 $) / V($ 育苗基质 $)=1 / 2]$ 至 $3 / 4$ 处, 直接播种 上述六种杂草靶标(芽率 $\geqslant 85 \%$ ), 覆土 $0.2 \mathrm{~cm}$, 待杂草 长至 3 叶期左右备用. 化合物 9a $\sim 9 x$ 以及商品化的对照 药剂硝磺草酮按照 $150 \mathrm{~g}$ ai/ha 剂量在自动喷雾塔施药 后, 待杂草叶面药液晾干后移入温室培养, 20 天后调查 结果.

\section{4 化合物作物选择性测试}

所合成的化合物采用盆栽法测试它们的作物选择 性: 供试靶标为玉米、大豆、棉花、水稻、大麦和油菜. 取内径 $7.5 \mathrm{~cm}$ 花盆, 装复合土 $[V$ (菜园土) $/ V$ (育苗基质) $=1 / 2$ ]至 $3 / 4$ 处, 直接播种上述六种作物靶标(芽率 $\geqslant$ $85 \%$ ), 覆土 $1.0 \mathrm{~cm}$, 待靶标长至 3 4 叶左右备用. 各化 合物按照 $150 \mathrm{~g}$ ai/ha 剂量在自动喷雾塔(型号: $3 \mathrm{WPSH}-700 \mathrm{E})$ 施药后, 待作物叶面药液晾干后移入温室 培养, 15 天后调查植株抑制率(\%), 确定药剂的作物安 全性.

\section{5 目标化合物 $9 a \sim 9 x$ 的体外 AtHPPD 酶抑制活性 测试}

偶联法测 $A t \mathrm{HPPD}$ 酶抑制活性: $A t \mathrm{HPPD}$ 表达，纯化 方法见参考文献 ${ }^{[8]}$. 对羟苯基丙酮酸(HPPA)在 HPPD 的 作用下转化生成尿黑酸(HGA), 而尿黑酸和底物 HPPA 的紫外吸收重叠且没有特征吸收, 无法将二者用紫外的 方法区分开来. 因此, 我们向体系中加入了另外一种酶 一尿黑酸双加氧酶(HGD)来辅助测试. HGD 可以将 HGA 催化转化成马来酰乙酰乙酸(MAA), 而后者在 318 $\mathrm{nm}$ 紫外处吸光度随着时间的变化而变化. 酶反应动力 学测试在 96 孔板中进行, 反应体系在 $\mathrm{pH}=7.0$ 的 HEPES buffer 中进行. 总体系共 $200 \mu \mathrm{L}: \mathrm{pH}=7.0$ 的 HEPES buffer, $2 \mathrm{mmol} / \mathrm{L}$ 抗坏血酸钠, $100 \mu \mathrm{mol} / \mathrm{L} \mathrm{FeSO}_{4}$, 
$1 \mu \mathrm{mol} / \mathrm{L}$ 抑制剂, 适量的 HPPD 和 HGD. 所测得化合 物的抑制剂常数 $K_{\mathrm{i}}$ 通过 Dixon 作图法求得.

\section{6 化合物 $9 i$ 和 $9 t$ 的分子对接方法}

通过 Sybyl-X 1.2 来构建 9i 和 9t, 并对它们赋予 Tripos 力场和 Gasteiger-Huckel 电荷, 采用 Powell 方法 优化 1000 步, 收玫标准设为 $0.005 \mathrm{kcal} /(\mathrm{mol} \bullet \AA ̊ 丿)$. 采用种 属来源为 $A t \mathrm{HPPD}$ 受体晶体结构(PDB code:1SQD), 剔 除结晶中的水分子和其它有机小分子, 保留参与络合的 二价铁, 并对受体加氢. 使用 Gold 3.2 进行分子对接, 将 $1 \mathrm{SQD}$ 中金属铁原子为中心 $15 \AA$ 半径内的区域设置 为活性腔格点扫描区，设定小分子与金属原子间形成配 位作用. 采用 GoldScore 进行打分, 其它位置保持默认 设置, 每个分子产生 100 个对接构象, 并分别取各自的 最优打分构象作为其代表的结合模式. 9i 和 $9 \mathrm{t}$ 与 $A t \mathrm{HPPD}$ 的结合模式均采用 Pymol 1.3 作图. 红色虚线代 表金属铁原子与周围氨基酸残基 His205, His287, Glu363 和小分子之间的络合作用以及小分子 $9 \mathrm{i}$ 与 Gln272 之间的氢键相互作用，灰色虚线代表抑制剂小 分子与周围氨基酸残基之间的立体冲撞效应.

\section{References}

[1] Dayan, F. E.; Duke, S. O.; Sauldubois, A.; Singh, N.; McCurdy, C.; Cantrell, C. Phytochemistry 2007, 68, 2004.

[2] Neidig, M. L.; Decker, A.; Choroba, O. W.; Huang, F. L.; Kavana, M.; Moran, G. R.; Spencer, J. B.; Solomon, E. I. Proc. Natl. Acad. Sci. 2006, 103, 12966.

[3] Kovaleva, E. G.; Lipscomb, J. D. Nat. Chem. Biol. 2008, 4, 186.

[4] Brownlee, J. M.; Johnson-Winters, K.; Harrison, D. H.; Moran, G. R. Biochemistry 2004, 43, 6370.

[5] Ahrens, H.; Lange, G.; Müller, T.; Rosinger, C.; Willms, L.; van Almsick, A. Angew. Chem., Int. Ed. 2013, 52, 9388.

[6] Beaudegnies, R.; Edmunds, A. J. F.; Fraser, T. E. M.; Hall, R. G.; Hawkes, T. R.; Mitchell, G.; Schaetzer, J.; Wendeborn, S.; Wibley, J. Bioorg. Med. Chem. 2009, 17, 4134.

[7] Zhang, Y. B. Mod. Agrochem. 2013, 12, 5. (张一宾, 现代农药, 2013, 12, 5.)

[8] Zhou, Y. Y.; Li, Z. M. World Pest. 2013, 35, 1. (周蕴望，李正名, 世界农药, 2013, 35, 1.)

[9] Nie, K. C.; Fan, Z. J.; Liu, C. L. Chin. J. Pest. 2006, 45, 4. (聂开晟, 范志金, 刘长令, 农药, 2006, 45, 4.)

[10] Lin, J.; Li, Z. G.; Zou, J. W.; Lu, S. Y. Acta Chim. Sinica 2012, 70, 1309. (林军, 李祖光, 邹建卫, 陆绍永, 化学学报, 2012, 70, 1309.)
[11] Zhu, Y. Q.; Si, X. K.; Zou, X. M.; Liu, B.; Yang, H. Z. Chin. J. Org. Chem. 2007, 27, 385. (朱有全, 司学凯, 邹小毛, 刘斌, 杨华铮, 有机化学, 2007, 27, 385.)

[12] Zhu, Y. Q.; Hu, F. Z.; Zou, X. M.; Yao, C. S.; Liu, B.; Li, Y. H.; Yang, H. Z. Chin. J. Org. Chem. 2005, 25, 419. (朱有全, 胡方中, 邹小毛，姚昌盛，刘斌，李永红，杨华铮，有机化学，2005，25, 419.)

[13] Huang, M. L.; Shang, Z. C.; Zou, J. W.; Yang, D. Y.; Yu, Q. S. Acta Chim. Sinica 2002, 60, 1558. (黄美兰, 商志才, 邹建卫, 杨定亚, 俞庆森, 化学学报, 2002, 60, 1558.)

[14] Wang, D. W.; Lin, H. Y.; Cao, R. J.; Ming, Z. Z.; Chen, T.; Hao, G. F.; Yang, W. C.; Yang, G. F. Pest Manag. Sci. 2014, doi: 10 1002/ps. 3894.

[15] Wang, D. W.; Lin, H. Y.; Cao, R. J.; Sheng, G. Y.; Chen, Q.; Hao, G. F.; Yang, W. C.; Yang, G. F. J. Agric. Food Chem. 2014, 62, 11786.

[16] Lee, D. L.; Prisbylla, M. P.; Cromartie, T. H.; Dagarin, D. P.; Howard, S. W.; Provan, W. M.; Ellis, M. K.; Fraser, T.; Mutter, L. C. Weed Sci. 1997, 45, 601.

[17] Takabe, F.; Fukumoto, S.; Kajiki, R.; Asakura, S.; Ueno, R.; Kobayashi, M.; Takahashi, S.; Yonekura, N.; Hanai, R.; Mitsunari, T. WO 2007088876 A1, 2007 [Chem. Abstr. 2007, 147, 874408].

[18] Tamai, R.; Ito, M.; Kobayashi, M.; Mitsunari, T.; Nakano, Y. WO 2009016841A1, 2009 [Chem. Abstr. 2009, 150, 138905].

[19] Takable, F.; Hirano, Y.; Funyu, A.; Kobayashi, M.; Mitsunari, T. WO 2010089993 A1, 2010 [Chem. Abstr. 2010, 153, 1001443].

[20] Patel, K. M.; Selby, T. P.; Smith, B. T.; Taggi, A. E.; Kovacs, P. R. WO 2012033548 A2, 2012 [Chem. Abstr. 2012, 156, 380307].

[21] Yang, W. C.; Lin, H. Y.; Yang, S. G.; Liu, W. N.; Yang, G. F. Chin. J. Pest. Sci. 2013, 15, 129. (杨文超, 林红艳, 杨盛刚, 刘万年, 杨 光富, 农药学学报, 2013, 15, 129.)

[22] Yang, W. C.; Li, H.; Zhu, X. L.; Wang, F.; Yang, G. F. ChemBioChem 2012, 13, 1542.

[23] Zhang, M. Z.; Chen, Q.; Mulholland, N.; Beattie, D.; Irwin, D.; Gu, Y. C.; Yang, G. F.; Clough, J. Eur. J. Med. Chem. 2012, 53, 283.

[24] Zhu, X. L.; Wang, F.; Li, H.; Yang, W. C.; Chen, Q.; Yang, G. F. Chin. J. Chem. 2012, 30, 1999.

[25] Jiang, L. L.; Zuo, Y.; Wang, Z. F.; Tan Y.; Wu, Q. Y.; Xi, Z.; Yang, G. F. J. Agric. Food Chem. 2011, 59, 6172.

[26] Chen, C. N.; Lv, L. L.; Ji, F. Q.; Chen, Q.; Xu, H.; Niu, C. W.; Xi, Z.; Yang, G. F. Bioorg. Med. Chem. 2009, 17, 3011.

[27] Chen, Q.; Liu, Y. C.; Zhang, M. Z.; Yang, G. F. Chin. J. Pest. Sci. 2009, 11, 31. (陈琼, 刘玉超, 张明智, 杨光富, 农药学学报, 2009, 11, 31.)

[28] Zhao, P. L.; Wang, F.; Zhang, M. Z.; Liu, Z. M.; Huang, W.; Yang, G. F. J. Agric. Food Chem. 2008, 56, 10767.

[29] Luo, Y. P.; Jiang, L. L.; Wang, G. D.; Chen, Q.; Yang, G. F. J. Agric. Food Chem. 2008, 56, 2118.

[30] Chen, Q.; Zhu, X. L.; Jiang, L. L.; Liu, Z. M.; Yang, G. F. Eur. J. Med. Chem. 2008, 43, 595.

[31] Li, Y. X.; Luo, Y. P.; Xi, Z.; Niu, C. W.; He, Y. Z.; Yang, G. F. J. Agric. Food Chem. 2006, 54, 9135.

[32] Chen, Q.; Long, D. Q.; Cheng, J.; Li, J.; Liu, Z. M.; Yang, G. F. Chem. J. Chin. Univ. 2006, 27, 454. (陈琼, 龙德清, 程靖, 李晶, 刘祖明, 杨光富, 高等学校化学学报, 2006, 27, 454.) 